\title{
Impact Factors in Nuclear Medicine Journals
}

\author{
Geoffrey M. Currie and Janelle M. Wheat \\ School of Biomedical Sciences, Charles Sturt University, Wagga Wagga, Australia
}

The annual Journal Citation Reports includes several statistics and figures, the key figure being journal impact factor. Journal impact factor, despite being widely used, is not well understood. This article aims to provide a broader understanding of impact factors and the implications thereof.

Key Words: impact factor; journal quality; nuclear medicine; academic

J Nucl Med 2007; 48:1397-1400

DOI: 10.2967/jnumed.107.041491

$\mathbf{J}$ ournal impact factor was introduced to replace the science citation index because the latter was biased toward journals with a large number of published articles (1). The impact factor allowed the indexing of smaller but influential journals (1). Certainly, nuclear medicine journals published quarterly would not have been included in the science citation index but might rank strongly with impact factors.

Thomson Scientific, or the Institute of Scientific Information (ISI) in a previous iteration, publishes Journal Citation Reports (JCR) annually. Although the JCR includes several statistics and figures, the key figure published is the journal impact factor. The impact factor, despite being widely used, is not well understood.

\section{WHAT IS THE IMPACT FACTOR?}

Journal impact factor is a measure of the ratio of recent citations to recent articles in the journal. Although providing the most comprehensive citation data for the evaluation of quality (2), the ISI $J C R$ should be interpreted with caution. The 2005 JCR indexed 6,088 journals-a 32\% increase over 10 y (2).

The ISI $J C R$ may be used for promotion, recruitment, or grant decisions. Identifying a candidate's publication record against the respective impact factor of journals is not so much an attempt to determine the quality of the journal as an attempt to gauge the quality of the individual publication. Generally, however, only a few articles contribute to the

\footnotetext{
Received Mar. 6, 2007; revision accepted Apr. 17, 2007.

For correspondence or reprints contact: Geoffrey M. Currie, PhD, School of Biomedical Sciences, Locked Bag 588, Charles Sturt University, Wagga Wagga 2678, Australia.

E-mail: gcurrie@csu.edu.au

COPYRIGHT @ 2007 by the Society of Nuclear Medicine, Inc.
}

overall impact factor of a journal, whereas most articles are either not or infrequently cited. This may be referred to as the $80 / 20$ phenomenon, where $80 \%$ of citations are attributed to $20 \%$ of articles (1). Journal impact factor alone, therefore, provides little indication of the impact or quality of a given article.

Journal impact factors should be interpreted with great care and with the acknowledgment that it represents only a rudimentary guide to journal quality. Even journals ranking among the highest in impact factor maintain a degree of skepticism regarding the usefulness of impact factors. The impact factor is not easily transferable or comparable across ISI categories. A high impact factor reflects simply the rate of citations to recent articles; it does not imply any degree of quality. A poorly written or methodologically flawed article is likely to attract numerous citations and contribute significantly to the impact factor of the journal.

In short, impact factor is the number of times the "average" article in a journal over the preceding 2 y was cited in journals in the subsequent year. That is, the 2005 impact factor is based on the number of citations in all ISI listed journals in 2005 of articles published in the specific journal in 2003 and 2004: Impact factor (2005) equals cites in 2005 to articles from 2003 and 2004, divided by number of articles in 2003 and 2004.

\section{SHORTCOMINGS OF IMPACT FACTORS}

ISI purports that the $J C R$, including impact factors, is an indicator of the frequency with which current researchers use a particular journal (3). One might imagine that this indicator is a poor one, given that:

- The reports are based on citations over a 2-y cycle.

- Publications in any single year may have been undertaken early in the 2-y cycle, and thus, initial analysis of existing literature may predate the 2-y cycle. That is, published work with an immediate "impact" on clinical practice or other researchers may have a less than indicative contribution to impact factor calculations.

- Cutting edge or innovative research may require a longer cycle before even those with rapid adoption generate publications citing the original work.

- The impact factor does not measure the longer citation life and prolonged high impact of seminal works. 
- Lack of citation does not indicate a lack of impact on either the research or the clinical practice of others.

- The JCR cycle is biased toward industries with rapidly evolving technology and techniques (e.g., information technology), while being biased against industries with a less dynamic evolution (e.g., mathematics).

Indeed, the very nature of health-based research handicaps health-based journals, for which the research cycle, including the relevant ethics approvals, radiation safety approvals, patient recruitment, and long sampling interval required for a measurable outcome, is significantly longer than that of the laboratory-based or field observational studies typical of many of the high-impact-factor science journals.

Nonetheless, impact factors might reflect the visibility or profile of a journal within a discipline. It has been suggested that the impact factor is biased toward North American journals and against non-English language journals (4). Mueller et al. (5), however, on investigating this proposition, found evidence only of a language bias, with no differences in impact factor noted between English language journals originating in the United States and those originating in other countries. The language bias is not entirely surprising given the predominance of English language journals in the ISI database and the inclination of individuals to rely more heavily on articles not requiring translation. Despite this, the German language nuclear medicine journal, Nuklearmedizin, has consistently performed strongly in the annual $J C R$ - perhaps a reflection of an active German-based nuclear medicine research community supporting a local journal and demonstrating a micro-level language bias.

Interpreting trends in impact factors needs careful consideration. One suspects that, across many disciplines, new technology and innovation emerge in waves. The cycle might reflect rapid adoption after release, a hiatus in advances as capabilities are fully realized, a lag phase as shortcomings are addressed, and a spike as solutions and advances are released. Journal impact factors might reflect this cycle. Interestingly, these cycles may be interrelated across disciplines. For example, the emergence of PET/CT may have boosted the impact factor of nuclear medicine journals, but as the technology became more broadly adopted clinically the influence of PET/CT may have extended to oncology journals, although one suspects that PET/CT articles in oncology journals might widely cite nuclear medicine journal articles, thus boosting the nuclear medicine impact factor.

\section{THE IMPACT FACTOR GAME}

Publishing one's research plays an important role in completing the project but also in disseminating the results to a target audience. Unfortunately, the role of impact factors in employment, promotion, and the like has shown a trend in which the journals with the highest impact factor are targeted for publication even when an alternative journal might provide broader dissemination to the target audience. This trend may perpetuate a downward cycle for those journals with low or no impact factor as fewer authors seek to publish articles in those journals or article quality decreases, reducing citations and reducing the impact factor. The converse argument might be that publications targeted toward the broadest valid audience might result in more citations. The Journal of Nuclear Medicine Technology (JNMT) provides a good example of the ideal outlet for dissemination of technically based research, but JNMT does not have an ISI listing. The absence of an ISI impact factor may be reflected in a change in the popularity of JNMT as an outlet for research. In the 4-y period 2003-2006, the average number of published articles in JNMT was 23.75 annually. In the 4-y period 1997-2000, a period before the emphasis on impact factors began, the average number of published articles in JNMT was 38.5 annually. Does this decline reflect a change in popularity, a change in editorial policy, or the emergence of impact factor-driven journal selection?

The role of the impact factor is not to guide decision making with regard to either the quality or the impact of individual articles. Because the central goal of publication is to disseminate findings to as broad a valid audience as possible, the number of times a journal article is actually readalthough a little more difficult to reliably measure-might better indicate impact. From an educator's perspective, what greater impact can a journal article have than if it is used to teach the future practitioners of that discipline?

A potential problem with the impact factor is the difficulty of comparing small and large disciplines. One might argue that high impact factors cannot be achieved in categories that have few researchers because the number of citations will be lower. That argument might be true if the same number of journals existed in each category. In reality, smaller disciplines or those with less research invariably have fewer journals and fewer active researchers. Nonetheless, the impact factor should not be used to directly compare disciplines or specialties.

In 2005, Ultrasonic Imaging had an impact factor of 0.938, similar to that of Clinical Physiology and Functional Imaging (0.973). The former value was based on just 9 published articles for the sampling period, whereas the latter was based on 57. The variations outside disciplines are more dramatic. In 2005, the highest ranked impact factor was that of Cancer: A Cancer Journal for Clinicians (49.794), based on just 20 published articles over 2 y. The Journal of Nuclear Medicine (JNM), which is arguably the most influential journal in nuclear medicine, had an impact factor of just 4.684, based on 294 articles. The impact factor is valid only within ISI categories, not between them (2).

\section{STRATEGIES TO BOOST IMPACT FACTORS}

Differences in policies on references may contribute to variations in impact factors between otherwise-similar journals. $J N M$, for example, limits the number of references per manuscript to 40 . A limit of 30 references is typical of many journals. Many other journals, including nuclear medicine journals such as Nuclear Medicine Communications, do 
not set a limit on the number of references. Although increasing the number of references per article contributes significantly to boosting the collective impact factor of a given discipline, removal of reference capping may not change the referencing practices of the average author. In 2005 , the mean number of references per article for JNM was 31.4 (3), which is well short of the limit of 40. Moreover, Nuclear Medicine Communications had an average of 22.5 references per article for 2005, despite having no capping policy. Perhaps removal of reference capping will only encourage those inclined toward gratuitous self-citation.

Self-citation is the practice of referencing one's own previously published work and, when used excessively, may bring into question the validity or quality of a manuscript. Self-citation may not always be limited by a reference-capping policy. For example, a publication of Clasen and Kulicke (6) contains 29 references, of which 23 (79\%) are self-references. Expanding the reference limit beyond 40 for $J N M$ might boost the impact factor of the journal and of the collective category on the ISI JCR. Although self-citation might then be encouraged, it is not always inappropriate or indulgent. Indeed, it may be the only means to citing references to cutting-edge research or innovation within the ISI JCR sampling period. Germano et al., who research quantitative gated myocardial perfusion SPECT, offer an example of how innovations in such research may cause it to be disproportionately represented in impact factor calculations. In one publication, Germano et al. (7) included 30 references, of which $18(60 \%)$ referred to work previously published by one of the authors. Thus, self-citation may simply reflect the expertise of a group of authors in a particular domain. Nonetheless, selfcitation is also a means by which individuals can boost their own citation index. Eugene Garfield is credited with the introduction of the science citation index and impact factor, and his recent brief communication (1) on the history of the impact factor contained $33.3 \%$ self-references. Removal of reference capping by one or more nuclear medicine journals may offer a strategy to increase the impact factor of the "radiology, nuclear medicine, and medical imaging" category compared with other categories but also to increase the impact factor of nuclear medicine journals relatively within the category.

Review articles generally attract a greater number of references than other types of articles, boosting the collective impact factor of the discipline. Review articles also tend to attract more citations than do original research articles. Thus, a journal based entirely on review articles, such as Seminars in Nuclear Medicine, might acquire an impact factor that, relative to a competing research journal such as $J N M$, does not reflect either quality or visibility. In the $J C R$ year of 2005, JNM included 289 articles but only 5 reviews (3), although JNM's own classification of a review article might be much higher. Increasing the number of reviews might boost the JNM impact factor relative to other nuclear medicine journals and to those in other categories.
There has been a proliferation of open-access journals in recent years. A typical PubMed query will retrieve any number of free full-text articles. Open access is a strategy being used to boost impact factors with some success because online availability of full-text articles increases the visibility of a journal (8). Many journals, such as JNM, rely on subscriptions, making an open-access strategy difficult to justify. Several journals offer open access to articles several years old while keeping the current volume closed to nonsubscribers. Clearly, this policy does not help improve the impact factor because the open access falls outside the JCR 2-y cycle.

Perhaps a more astute approach might be to offer selective open access to the current volume. The status and profile of $J N M$ is based in a large part on the quality and cutting-edge nature of the research published. The continuing education articles and reviews not only represent a small proportion of published work but also fall outside the core competitive advantage of JNM. Seminars in Nuclear Medicine, on the other hand, centers on review articles. Review articles do, however, attract more frequent citations in other journal articles and, thus, contribute more to journal impact factors. $J N M$ might boost its impact factor without losing subscriptions by providing all review articles as open access online while maintaining subscription-only access to other articles.

Several other strategies have been used with some success to improve the impact factor of a journal:

- Publication in multiple languages, particularly when the native language is not English, expands readership and the potential for citation.

- A selective editorial policy may be adopted in which articles are rejected or accepted with some consideration to the likelihood that they will be cited. An important factor to consider is that a controversial issue likely to divide a community may attract many citations.

- An astute choice of topics for journals, particular with respect to review articles, may increase the impact factor.

Although $J N M$ has dominated the impact factor rankings for nuclear medicine journals fairly consistently over the last $8 \mathrm{y}$, several other nuclear medicine journals have ridden the impact-factor roller coaster. Seminars in Nuclear Medicine offers the best example, with a fairly consistent ranking of third for nuclear medicine journals over $8 \mathrm{y}$. In 2003, Seminars in Nuclear Medicine had an impact factor of 3.431, which rose to 5.800 in 2004 and then fell to 3.000 in 2005. The rise probably reflected the interest of researchers in emerging techniques, including fresh themes for PET, lung CT (CT pulmonary angiography), peptides, and ${ }^{18}$ F-FDG. The subsequent drop probably reflected a lack of research interest due to the narrowing and ongoing theme of PET.

\section{JNMT}

Despite the 7,500 scholarly journals included in the ISI $J C R$, there are several omissions, most notably JNMT. It is 
crucial to recognize that journals with a low impact factor or, like JNMT, no ISI listing will continue to play an important role in the dissemination of new knowledge. Had JNMT been listed with ISI, the 2005 impact factor would have been 0.350 , with 44 articles over the $2-y$ period. This value corresponds to a rank of $82 / 85$ in the category and $5,458 / 6,088$ overall.

\section{CONCLUSION}

The ISI JCR needs to be interpreted with caution. The impact factor is a good rudimentary tool for illuminating trends in journal quality within disciplinary groups. The impact factor itself should be used as a guide rather than a tool for definitive decision making and is best interpreted in conjunction with the entire content of the JCR. Several strategies can boost the impact factor of a journal, but ironically, although impact factor may increase, a decrease in the actual quality of the journal may result. In the absence of a single instrument to measure the actual impact of a journal (or its contents) on policy and practice, the $J C R$ impact factor provides rankings reflective of the visibility of nuclear medicine journals.

\section{REFERENCES}

1. Garfield E. The history and meaning of the journal impact factor. JAMA. 2006; 295:90-93.

2. Andersen J, Belmont J, Cho CT. Journal impact factor in the era of expanding literature. J Microbiol Immunol Infect. 2006;39:436-443.

3. ISI Web of Knowledge. About journal citation reports. Available at: http:// admin.isiknowledge.com/JCR/help/h_jcrabout.htm. Accessed May 18, 2007.

4. Adams AB, Simonson D. Publications, citations, and impact factors of leading investigators in critical care medicine. Respir Care. 2004;49:276-281.

5. Mueller PS, Murali NS, Cha SS, Erwin PJ, Ghosh AK. The association between impact factors and language of general internal medicine journals. Swiss Med Wkly. 2006;136:441-443.

6. Clasen C, Kulicke WM. Rheo-optical and rheo-mechanical characterization of the viscoelastic properties and shear-induced orientation of hydrocolloids. In: Fischer P, Marti I, Windhab EJ, eds. Proceedings of the 2nd International Symposium on Food Rheology and Structure. Zurich, Switzerland: ETH Zurich; 2000:25-33.

7. Germano G, Kavanagh PB, Waechter P, et al. A new algorithm for the quantitation of myocardial perfusion SPECT. I: Technical principles and reproducibility. J Nucl Med. 2000;41:712-719.

8. Mueller PS, Murali NS, Cha SS, Erwin PJ, Ghosh AK. The effect of online status on the impact factors of general internal medicine journals. Neth J Med. 2006; 64:39-44. 